\title{
Effect of total flavonoids of Epimedium on climacteric depression model
}

\author{
Mingsan Miao ${ }^{1, a,{ }^{*}}$, Peng Xi ${ }^{2, b}$, Bolin Cheng ${ }^{3, c}$, Mengfei Zhang ${ }^{4, d}$, \\ Tisheng $\mathrm{Cui}^{5, \mathrm{e}}$
}

\author{
1,2,3,4 Henan University of Traditional Chinese Medicine, Zhengzhou, 450008, china \\ aemail:miaomingsan@163.com, bemail:752456276@qq.com, cemail:2209243431@qq.com, \\ demail:457291457@qq.com, eemail:869122320@qq.com, ”corresponding author
}

\begin{abstract}
Keyword: total flavonoids of Epimedium; climacteric depression model; Gengnian'an capsule group; Soybean isoflavone group
\end{abstract}

Abstract: Objective:To study effect of total flavonoids of Epimedium on climacteric depression model. Methods: In addition to the blank group, other rats climacteric models, total removal of the left ovary, the right ovary removed $80 \%$. After the injection of penicillin to prevent infection, continuous injection 3 days, operation started by only for vaginal smear of rats after $5 \mathrm{~d}$, one times a day, a continuous smear 5 days, smear showing estrous response of the animal discard without, selection of animal 60 castrated completely random divided into 6 groups for the experiment. After two weeks of administration, the normal group was not given any stimulus, to make the model of group of single cage monoculture, everyday to given different stimulation. Result: The total flavonoids of Epimedium large, middle, small dose can significantly improve the depressive rats $24 \mathrm{~h}$ sucrose intake, horizontal and vertical integral of motion, levels of 5-HT and DA in brain homogenate, thymus index, the uterus index, the level of serum E2, The decrease of levels of serum LH, FSH, the elevation of serum IL-2, to improve the content of BGP in serum. Conclusion: The total flavonoids of Epimedium each dose of each index of perimenopausal depression model rats have avery good improvement.

\section{Introduction}

Women menopause depression is a mood disorder that is most common in old age the transition stage from sexual maturity, the incidence rate is rising year by year. The present research work mainly focus on the psychological and social factors, biochemical, neuroendocrine etc. The experiment adopts ovariectomy and chronic unpredictable stress combination, two-step method produced an animal model of depression menopause, aims to further explore the pathogenesis of the disease and provide an experimental basis for the screening of effective drugs [1].

\section{Materials}

Animal

Wistar rats, female, $160 \sim 200 \mathrm{~g}$, provided by medical experimental animal center of Hebei Province, Certificate No.: 701022.

\section{Experimental drug}

Epimedium extract (epimedium total Huang Tong), Xi'an City Park days biological preparation factory, batch number: TY200080116; Gengnian an Capsules, Changchun Yingping Pharmaceutical Co., Ltd. batch number: 20070521. Times Nuokang soy isoflavone tablets, Nanning fresh life Biological Technology Co., Ltd. Southern food certificate (2006) No. 450101-000389

\section{Reagents and instruments}

Pentobarbital sodium, Chinese Pharmaceutical Group Shanghai Chemical Reagent Company, batch number: F20030816; Formaldehyde, China Laiyang Shuangshuang Chemical Co. Itd., batch number: 20040519; Benzylpenicillin sodium for injection, North China Pharmaceutical Limited by Share Ltd, batch number: X0603113; Phthalaldehyde, Shanghai chemical reagent purchasing supply Wulian chemical plant production, batch number: 20060101, Butanol, Tianjin city Kaitong 
Chemical Reagent Co., Ltd production, batch number: 050105; Heptane, Tianjin city four chemical plant, DA, Sigma company; Sodium periodate, Tianjin Fuchen Chemical Reagent Factory production, Lot: 20021105; EDTA-2Na, Baosight biotechnology limited company production, Lot: 20051021; Two sodium hydrogen phosphate, Tianjin city Kaitong Chemical Reagent Co., Ltd production, Lot 20051212; Sodium acetate, Tianjin Chemical Reagent Factory Production, Lot 20030808; Sodium sulfite, Tianjin city Kaitong Chemical Reagent Co., Ltd production, Lot 20051108; Iodine, Tianjin Kermel Chemical Reagent Co., Ltd production, Lot 20050405; Estradiol radioimmunoassay kit, Beijing Kemei Leya Biological Technology Co., Ltd., Batch: the country medicine accurate S20063125; Luteinizing hormone radioimmunoassay kit, Beijing Kemei Leya Biological Technology Co., Ltd., Batch: the country medicine accurate S20063073; Follicle stimulating hormone radioimmunoassay kit, Beijing Kemei Leya Biological Technology Co., Ltd., Batch: the country medicine accurate S20063072; Interleukin -2 radioimmunoassay kit, Beijing S \& P Weiye Biological Technology Co. 1td.; Osteocalcin radioimmunoassay kit, Beijing S \& P Weiye Biological Technology Co. ltd.; SN-695 B intelligent radioimmunoassay gamma survey meter, Shanghai nuclear instrument factory Research Institute of ring; F-4500 fluorescence spectrophotometer, Hitachi company.

\section{Methods}

\section{Modeling and Administration}

Modeling method: Take 80 Wistar rats, female,160 200g, Were randomly divided into 7 groups, divided into blank control group, model group, Gengnian'an capsule group, soybean isoflavone group, total flavonoids of Epimedium high, medium and low dose group. In addition to the control group, the rats were intraperitoneal injection of pentobarbital sodium $50 \mathrm{mg} / \mathrm{kg}$ after anesthesia abdominal position fixing, then from the rat back last rib, about $2 \mathrm{~cm}$ in the axillary line at the intersection of shearing away from the spine and the outside, after disinfection skin incision and back muscles about $1.5 \sim 2 \mathrm{~cm}$, in the view of visible incision creamy white shiny cellulite, ovary is embedded. Using tweezers to gently pull the incision outside grip cellulite, separation of cellulite, can see a group irregularly shaped thin red yellow ovary. Under the first ovarian tubal clipping (including fat) with a thin ligature, complete removal of the left ovary, the right of removal of $80 \%$, postoperative uterine horn back homeopathy in abdominal cavity, suturing muscle and skin, after careful breeding, injection of penicillin to prevent infection, continuous injection of 3 days, operation started by only of rat vaginal smears after $5 \mathrm{~d}, 1$ times a day, consecutive of $5 \mathrm{~d}$, smear showing emotional reactions animal was discarded, selection of animal 60 castrated completely random divided into 6 groups for the experiment. After two weeks of administration, the normal group each 5 cage rearing, normal diet and water, don't give any stimulation, randomly assigned to different stimuli every day. The 7 kind of stress factor according to the random method is used in the 20d: (1) night lighting (24h); (2) fasting (24h); (3) water deprivation (24h); (4) 4 C swimming in cold water for 5 minutes; (5) 1 minute clip tail; (6) 5 minute level oscillations; (7) $2 \mathrm{~h}$ behavioral restrictions. Randomized to receive a stimulus every day, each stimulus cannot appear continuously, continuous of $20 \mathrm{~d}$.

\section{Open field test method}

The experimental device is a cubic box open, high of $40 \mathrm{~cm}$, length and width of $80 \mathrm{~cm}$, the peripheral wall and the bottom surface are black, the bottom surface is composed of 25 blocks of equal area composition, divided by white lines. During the experiment the rats were placed in the center of the box open box, rats were observed within 30 minutes across the bottom surface of the block number (box only count four claws are entering) the level of activity (crossing) score, and the rear up times (two forepaw vacated or climb the walls) vertical activity (rearing) score. After each experiment shall completely remove manure, each animal was measured 1 times at 2 hours after administration, comparison of the difference scores of each group. This experiment was conducted in a quiet room. 


\section{Effects on monoamine neurotransmitters in the brain}

The rats were sacrificed after taking blood, the brain, brain tissue preparation, determination of content of brain tissue homogenate of monoamine neurotransmitter 5-HT, DA by fluorescence method.

Brain tissue removal of blood film, the olfactory bulb and the cerebellum, and then weighing, with 5 times amount of acidified butanol (n-butanol with $1000 \mathrm{~mL}$ adding concentrated hydrochloric acid $0.85 \mathrm{~mL}$ ) were homogenized in an ice water bath. Vortex $5 \mathrm{~min}$, centrifuge $5 \mathrm{~min}$ (3000 rev / min). The supernatant was $2.5 \mathrm{~mL}$, into another centrifuge tube with stopper, plus $\mathrm{n}$-heptane $5 \mathrm{~mL}$ and $0.1 \mathrm{~mol} \cdot \mathrm{L}^{-1} \mathrm{HCl} 1.2 \mathrm{~mL}$, vortex $5 \mathrm{~min}$, centrifuge $5 \mathrm{~min}$, to give a hierarchical solution (aqueous phase I containing of 5-HT, DA). 5-HT, DA standards were used $0.01 \mathrm{~mol} \cdot \mathrm{L}^{-1}$ hydrochloride formulated into $500 \mu \mathrm{g} \cdot \mathrm{mL}^{-1}$ as a standard stock solution, store in refrigerator $2{ }^{\circ} \mathrm{C}$, just before each take the storage of liquid $0.25 \mathrm{~mL}$, diluted with $0.01 \mathrm{~mol} \cdot \mathrm{L}^{-1}$ solution of hydrochloric acid to $100 \mathrm{~mL}$ standard applications, each standard concentration of $1.25 \mu \mathrm{g} \bullet \mathrm{mL}^{-1}$. Water phase I $0.5 \mathrm{~mL}$ adding $0.5 \%$ cysteine $\left(0.1 \mathrm{~mol} \cdot \mathrm{L}^{-1}\right.$ hydrochloric acid freshly prepared) $0.1 \mathrm{~mL}, 0.005 \%$ phthalaldehyde $3 \mathrm{~mL}$ (to $10 \mathrm{~mol} \cdot \mathrm{L}^{-1} \mathrm{HCl}$ temporarily dubbed), $0.02 \%$ sodium periodate $0.1 \mathrm{~mL}$, the boiling water bath for 10 minutes, cooled to room temperature, measurement. Standard pipe standard applying solution $0.5 \mathrm{~mL}$, take a blank tube re-distilled water $0.5 \mathrm{~mL}$, plus acidification butanol $4.5 \mathrm{~mL}$, vortex $5 \mathrm{~min}$, centrifuge $5 \mathrm{~min}(3000 \mathrm{rev} / \mathrm{min})$, supernatant was $2.5 \mathrm{~mL}$, into another centrifuge tube with stopper, plus $\mathrm{n}$-heptane $5 \mathrm{~mL}$ and $0.1 \mathrm{~mol} \cdot \mathrm{L}^{-1} \mathrm{HCl} 1.0 \mathrm{~mL}$, vortex $5 \mathrm{~min}$, centrifuge $5 \mathrm{~min}$, stratification was obtained (the aqueous phase I containing of 5-HT, DA). Water phase I $0.5 \mathrm{~mL}$ adding $0.5 \%$ cysteine $0.1 \mathrm{~mL}, 0.005 \%$ phthalaldehyde $3 \mathrm{~mL}$ (with $10 \mathrm{~mol} \cdot \mathrm{L}^{-1}$ $\mathrm{HCl}$ dubbed), $0.02 \%$ sodium periodate $0.1 \mathrm{~mL}$, the boiling water bath for 10 minutes, cooled to room temperature, measurement. The maximum emission wavelength of 5-HT(EM) is $475 \mathrm{~nm}$, the maximum excitation wavelength (ex) is $355 \mathrm{~nm}$.

Water phase I $0.5 \mathrm{~mL}$, add $1 / 15 \mathrm{~mol} \cdot \mathrm{L}^{-1}$ of phosphate buffered saline $(\mathrm{pH}=7.2) 1.7 \mathrm{~mL}, 0.1 \mathrm{~mol} \cdot \mathrm{L}^{-1}$ EDTA2Na (to $1 \mathrm{~mol} \cdot \mathrm{L}^{-1}$ sodium acetate solution formulated, to $10 \mathrm{~mol} \cdot \mathrm{L}^{-1}$ sodium hydroxide to adjust $\mathrm{pH}$ to 7.0 ) $0.4 \mathrm{~mL}$, Iodine reagent (in $70 \%$ ethanol configured to $5 \%$ sodium iodide solution, and $0.02 \mathrm{~mol} \cdot \mathrm{L}^{-1}$ iodine solution before use with a $1: 1$ volume ratio) $0.1 \mathrm{~mL}$, stand for 2 minutes, adding an alkaline sodium sulphite solution $0.5 \mathrm{~mL}(25 \%$ sodium sulfite $1.0 \mathrm{~mL}, 5 \mathrm{~mol} / \mathrm{L}$ sodium hydroxide $9 \mathrm{~mL}$ temporary mix), stand for 2 minutes, accession to the $6 \mathrm{~mol} \cdot \mathrm{L}^{-1}$ of acetic acid in liquid $0.5 \mathrm{~mL}$, theboiling water bath for 20 minutes, cooling to room temperature, DA was measured. The maximum emission wavelength of DA (EM) is $370 \mathrm{~nm}$, the maximum excitation wavelength (Ex) is $322 \mathrm{~nm}$.

Calculation formula: The content of monoamine neurotransmitters(brain tissue samples of ug $\cdot \mathrm{g}-1)=(\mathrm{Ri}-\mathrm{Rib}) /(\mathrm{Rr}-\mathrm{Rrb}) \times 0.5(\mathrm{~mL}) \times 1.25(\mathrm{ug} \cdot \mathrm{mL}-1) \div$ actual brain weight

$\mathrm{Ri}$ : as for sample fluorescence readings; Rib: as for sample blank fluorescence readings;

$\mathrm{Rr}$ : as the control fluorescence readings; Rrb: as the control blank readings.

\section{Statistical}

Data analysis for statistical data with statistical package SPSS 13.0 for windows, Measurement data with the mean \pm standard deviation $( \pm s)$ said, comparison between groups using analysis of variance (LSD method). 


\section{Results}

Effects of drugs on sugar consumption in rats Menopause Depression of the model (see Table 1).

Table 1 Effects of drugs on menopausal depression model rats 24 hours sugar intake $(\overline{\mathrm{X}} \pm \mathrm{s})$

\begin{tabular}{cccc}
\hline Groups & $\begin{array}{c}\text { number of animals } \\
\text { (only) }\end{array}$ & Dose (mg $\bullet$ kg-1) & consumption of sucrose (mL) \\
\hline Blank control group & 10 & - & $41.3 \pm 5.1$ \\
Model group & 10 & - & $20.9 \pm 2.8$ \\
Gengnian'an capsule group & 10 & 450 & $26.7 \pm 5.2 *$ \\
Soybean isoflavone group & 10 & 210 & $24.9 \pm 3.4 * *$ \\
total flavonoids of epimedium high dose group & 10 & 140 & $28.4 \pm 4.6 * *$ \\
total flavonoids of epimedium middle dose group & 10 & 70 & $27.5 \pm 2.0 * *$ \\
total flavonoids of Epimedium small dose group & 10 & $25.3 \pm 2.8 *$ \\
\hline
\end{tabular}

Note: Compared with the control group, ${ }^{\triangle} P<0.05,{ }^{\triangle \triangle} P<0.01$; Compared with the model group, ${ }^{*} P<0.05,{ }^{* *} P<0.01$

As can be seen from the table, compared with the control group, sucrose intake of 24 hours of model group was significantly decreased $(P<0.01)$, reflects the degree of response of rats to reduce reward. Compared with the model group, Gengnian'an capsule group and total flavonoids of Epimedium small dose group could be significantly improved depression in rats 24 hours of sugar intake $(P<0.05)$, total flavonoids of Epimedium middle、 high dose group and soybean isoflavone group could be significantly improved depression in rats 24 hours of sugar intake $(P<0.01)$. Effect of drug behavior of depression model rats with menopause (see Table 2)

Table 2 Effects of drugs on the level of Menopause Depression Model Rats - vertical integration ( $\overline{\mathrm{X}} \pm \mathrm{s})$

\begin{tabular}{|c|c|c|c|c|}
\hline Groups & $\begin{array}{l}\text { number of animals } \\
\text { (only) }\end{array}$ & Dose $(\mathrm{mg} \bullet \mathrm{kg}-1)$ & $\begin{array}{l}\text { Horizontal motion } \\
\text { score (points) }\end{array}$ & $\begin{array}{l}\text { Vertical motion score } \\
\text { (points) }\end{array}$ \\
\hline Blank control group & 10 & - & $144.6 \pm 23.6$ & $89.0 \pm 15.6$ \\
\hline Model group & 10 & - & $73.4 \pm 13.6^{\triangle \Delta}$ & $43.8 \pm 12.4^{\triangle \Delta}$ \\
\hline Gengnian'an capsule group & 10 & 450 & $110.8 \pm 19.3 * *$ & $70.6 \pm 16.0 * *$ \\
\hline Soybean isoflavone group & 10 & 85 & 119. $6 \pm 25.9 * *$ & $67.4 \pm 17.9 *$ \\
\hline $\begin{array}{l}\text { Total flavonoids of epimedium } \\
\text { high dose group }\end{array}$ & 10 & 210 & $148.8 \pm 20.0 * *$ & $73.5 \pm 18.5 * *$ \\
\hline $\begin{array}{l}\text { Total flavonoids of epimedium } \\
\text { middle dose group }\end{array}$ & 10 & 140 & $140.8 \pm 20.5 * *$ & $72.2 \pm 15.9 * *$ \\
\hline $\begin{array}{l}\text { Total flavonoids of } \\
\text { Epimedium small dose group }\end{array}$ & 10 & 70 & $99.8 \pm 13.3 * *$ & $60.4 \pm 11.6 *$ \\
\hline
\end{tabular}

Note: compared with the blank group, ${ }^{\triangle} P<0.05,{ }^{\triangle} P<0.01$; Compared with the model group, ${ }^{*} P<0.05,{ }^{* *} P<0.01$

As can be seen from the table, compared with the control group, horizontal, vertical integration of motion of model group were significantly reduced $(P<0.01)$, reflects the rats on the fresh environment reduced the degree of curiosity. Compared with the model group, Gengnian'an capsule, total flavonoids of Epimedium middle、 high dose group could significantly increase the rat horizontal and vertical integration of motion $(P<0.01)$, total flavonoids of Epimedium small dose group and soybean isoflavones could significantly improve the level ofintegration of motion $(P<0.01)$, significantly improve the vertical integral of motion $(P<0.05)$. 


\section{Effects of drugs on climacteric depression model rats in the levels of monoamine neurotransmitter}

Table 3 Effects of drugs on rat brain level of 5-HT, DA Menopause Depression model $(\overline{\mathrm{X}} \pm \mathrm{s})$

\begin{tabular}{ccccc}
\hline Groups & $\begin{array}{c}\text { number of animals } \\
\text { (only) }\end{array}$ & Dose $(\mathrm{mg} \bullet \mathrm{kg}-1)$ & 5-HT $(\mu \mathrm{g} / \mathrm{g}$ brain $)$ & DA $(\mu \mathrm{g} / \mathrm{g}$ brain $)$ \\
\hline Blank control group & 10 & - & $0.707 \pm 0.076$ & $0.696 \pm 0.040$ \\
Model group & 10 & - & $0.468 \pm 0.041^{\triangle \triangle}$ & $0.457 \pm 0.045^{\triangle \Delta}$ \\
Gengnian'an capsule group & 10 & 450 & $0.508 \pm 0.015^{*}$ & $0.529 \pm 0.058^{*}$ \\
$\begin{array}{c}\text { Soybean isoflavone group } \\
\text { Total flavonoids of epimedium } \\
\text { high dose group }\end{array}$ & 10 & 85 & $0.537 \pm 0.060^{* *}$ & $0.542 \pm 0.093^{*}$ \\
$\begin{array}{c}\text { Total flavonoids of epimedium } \\
\text { middle dose group }\end{array}$ & 10 & 210 & $0.568 \pm 0.033^{* *}$ & $0.579 \pm 0.051^{* *}$ \\
$\begin{array}{c}\text { Total flavonoids of } \\
\text { Epimedium small dose group }\end{array}$ & 10 & 140 & $0.524 \pm 0.064^{*}$ & $0.530 \pm 0.038^{* *}$ \\
\hline
\end{tabular}

Note: Compared with the control group, ${ }^{\triangle} P<0.05,{ }^{\triangle}{ }^{\triangle} P<0.01$; Compared with the model group, ${ }^{*} P<0.05,{ }^{* *} P<0.01$

As can be seen from the table, compared with the control group, model group in brain homogenate levels of 5-HT, DA were significantly reduced $(P<0.01)$, description made a successful rat model of chronic stress depression. Compared with the model group, Gengnian'an capsule, total flavonoids of epimedium middle dose group could significantly improve brain homogenates of 5-HT levels $(P<0.05)$, Soybean isoflavone group, total flavonoids of epimedium high dose group could significantly improve the levels $(P<0.01)$; Gengnian'an capsules, Soybean isoflavone group could significantly improve brain homogenates of DA levels $(P<0.05)$, middle and high doses group of Epimedium Flavonoids could significantly improve the levels $(P<0.01)$.

\section{Effects of drugs on organ index menopause with depression model rats}

Table 4 Effects of drugs on Menopause Depression Model of rat thymus, spleen, uterus index $(\overline{\mathrm{X}} \pm \mathrm{s})$

\begin{tabular}{|c|c|c|c|c|c|}
\hline Groups & $\begin{array}{c}\text { number of } \\
\text { animals (only) }\end{array}$ & Dose $\left(\mathrm{mg} \cdot \mathrm{kg}^{-1}\right)$ & Thymus index $\left(\mathrm{mg} \cdot \mathrm{g}^{-1}\right)$ & Spleen index $\left(\mathrm{mg} \cdot \mathrm{g}^{-1}\right)$ & $\begin{array}{l}\text { Uterine Index } \\
\left(\mathrm{mg} \cdot \mathrm{g}^{-1}\right)\end{array}$ \\
\hline Blank control group & 10 & - & $1.124 \pm 0.239$ & $2.613 \pm 0.352$ & $1.707 \pm 0.571$ \\
\hline Model group & 10 & - & $0.848 \pm 0.126^{\triangle \Delta}$ & $1.931 \pm 0.550^{\triangle}$ & $0.982 \pm 0.315^{\triangle \Delta}$ \\
\hline Gengnian'an capsule group & 10 & 450 & $1.060 \pm 0.397$ & $2.376 \pm 0.778$ & $1.004 \pm 0.277$ \\
\hline Soybean isoflavone group & 10 & 85 & $1.194 \pm 0.251 * *$ & $2.176 \pm 0.408$ & $1.346 \pm 0.419$ \\
\hline $\begin{array}{l}\text { Total flavonoids of epimedium high } \\
\text { dose group }\end{array}$ & 10 & 210 & $0.858 \pm 0.327$ & $1.969 \pm 0.597$ & $1.454 \pm 0.492 *$ \\
\hline $\begin{array}{l}\text { Total flavonoids of epimedium } \\
\text { middle dose group }\end{array}$ & 10 & 140 & $1.209 \pm 0.473$ & $2.580 \pm 0.528$ & $1.082 \pm 0.211$ \\
\hline $\begin{array}{c}\text { Total flavonoids of } \\
\text { Epimedium small dose group }\end{array}$ & 10 & 70 & $0.972 \pm 0.189$ & $2.579 \pm 0.742$ & $1.374 \pm 0.569$ \\
\hline
\end{tabular}

Note: Compared with the control group, ${ }^{\triangle} P<0.05,{ }^{\triangle \triangle} P<0.01$; Compared with the model group, $* P<0.05,{ }^{* *} P<0.01$

As can be seen from the table, compared with the control group, spleen index was significantly decreased in model group $(P<0.05)$, thymus index, uterine index was significantly decreased $(P<0.01)$, description of model rats with Involutional Depression in thymus, spleen and uterus tissue atrophy. Compared with the model group, soybean isoflavone group could significantly improve the thymus index of model animal $(P<0.01)$, High dose of total flavonoids of Epimedium could significantly improve the uterus index $(P<0.05)$.

Effects of drugs on serum sex hormones in rats model of menopause with depression

Table 5 Effects of drugs on the content of E2, FSH,LH in serum on perimenopausal depression model rats $(\overline{\mathrm{X}} \pm \mathrm{s})$

\begin{tabular}{|c|c|c|c|c|c|}
\hline Groups & $\begin{array}{c}\text { number of } \\
\text { animals (only) }\end{array}$ & Dose $\left(\mathrm{mg} \cdot \mathrm{kg}^{-1}\right)$ & $\mathrm{E}_{2}\left(\mathrm{pg} \cdot \mathrm{mL}^{-1}\right)$ & LH $\left(\mathrm{IU} \cdot \mathrm{L}^{-1}\right)$ & FSH $\left(I U \cdot L^{-1}\right)$ \\
\hline Model group & 10 & - & $10.56 \pm 1.24^{\triangle \triangle}$ & $9.705 \pm 1.303^{\triangle \triangle}$ & $4.287 \pm 0.806^{\triangle \Delta}$ \\
\hline Soybean isoflavone group & 10 & 85 & $13.34 \pm 1.70 * *$ & $7.998 \pm 1.109^{* *}$ & $3.446 \pm 0.237 *$ \\
\hline $\begin{array}{l}\text { Total flavonoids of epimedium high dose } \\
\text { group }\end{array}$ & 10 & 210 & $13.64 \pm 1.33^{* *}$ & $8.235 \pm 0.454 *$ & $3.194 \pm 0.404 * *$ \\
\hline $\begin{array}{c}\text { Total flavonoids of epimedium middle } \\
\text { dose group }\end{array}$ & 10 & 140 & $11.17 \pm 0.68$ & $9.077 \pm 0.520$ & $3.626 \pm 0.425 *$ \\
\hline
\end{tabular}

Note: Compared with the control group, ${ }^{\triangle} P<0.05,{ }^{\triangle \triangle} P<0.01$; Compared with the model group, ${ }^{*} P<0.05,{ }^{* *} P<0.01$ 
As can be seen from the table, compared with the control group, the level of serum E2 of model group decreased significantly $(P<0.01)$, levels of LH, FSH were significantly increased $(P<0.01)$, description of sex hormone in rat model of Menopause Depression Disorder. Compared with the model group, soybean isoflavone group, high doses of total flavonoids of Epimedium could significantly increase levels of serum E2 $(P<0.01)$; soybean isoflavone group $(P<0.01)$, high doses of total flavonoids of Epimedium $(P<0.05)$ were significantly and decrease the level of LH in serum; Gengnian'an capsule group, soybean isoflavone group and middle dose of total flavonoids of epimedium group could significantly reduce the level of FSH in serum $(P<0.05)$, high dose of total flavonoids of Epimedium could significantly decrease the level of FSH $\quad(P<0.01)$.

Effect of drugs on the IL-2, the BGP content of serum in perimenopausal depression model rats Table 6 Effect of drugs on the IL-2,the BGP content of serum in perimenopausal depression model rats

\begin{tabular}{|c|c|c|c|c|}
\hline Groups & number of animals (only) & Dose $\left(\mathrm{mg} \cdot \mathrm{kg}^{-1}\right)$ & IL-2（ng $\left.\cdot \mathrm{mL}^{-1}\right)$ & BGP $\left(\mu g \cdot L^{-1}\right)$ \\
\hline Blank control group & 10 & - & $2.908 \pm 0.656$ & $2.826 \pm 0.670$ \\
\hline Model group & 10 & - & $1.728 \pm 0.279^{\triangle \Delta}$ & $1.981 \pm 0.465^{\triangle \Delta}$ \\
\hline Gengnian'an capsule group & 10 & 450 & $2.452 \pm 0.303 * *$ & $2.587 \pm 0.669$ \\
\hline Soybean isoflavone group & 10 & 85 & $1.782 \pm 0.244$ & $2.253 \pm 0.416$ \\
\hline $\begin{array}{l}\text { Total flavonoids of epimedium high dose } \\
\text { group }\end{array}$ & 10 & 210 & $2.234 \pm 0.419 * *$ & $2.324 \pm 0.295 *$ \\
\hline $\begin{array}{l}\text { Total flavonoids of epimedium middle dose } \\
\text { group }\end{array}$ & 10 & 140 & $2.047 \pm 0.286^{*}$ & $2.310 \pm 0.438$ \\
\hline $\begin{array}{l}\text { Total flavonoids of Epimedium small dose } \\
\text { group }\end{array}$ & 10 & 70 & $1.851 \pm 0.349$ & $2.138 \pm 0.299$ \\
\hline
\end{tabular}

Note: compared with the blank group, ${ }^{\triangle} P<0.05,{ }^{\triangle \triangle} P<0.01$; Compared with the model group, ${ }^{*} P<0.05, * * P<0.01$

As can be seen from the table, compared with the blank group, levels of IL-2, BGP serum were significantly lower in model group $(P<0.01)$, description of rats with Involutional Depression immunity decreased and osteoporosis. Compared with the model group, Gengnian'an capsules, high dose of total flavonoids of Epimedium could be significantly increased serum levels of IL-2 $(P<0.01)$, middle dose of total flavonoids of Epimedium could significantly increase the level of IL-2 $(P<0.05)$, high dose of total flavonoids of Epimedium could obviously increase the content of BGP in serum $(P<0.05)$.

\section{Discussion}

Climacteric depression belongs to the Chinese medicine in the "melancholia", "hysteria", "lily disease" and "menopause syndromes" category, is one of the clinical common disease, frequently occurring disease. The main pathogenesis of disease is "kidney liver stagnation, phlegm by God," that God awakened unclear, Lesions involving the heart, kidney, liver, spleen, brain [2].

The current commonly used menopausal syndrome animal model with two kinds of: Ovariectomized animal model, natural menopause animal models. Model used in the production of most animals are rats, mice, because of its relatively close to human physiological condition, and cheap [3]. $90 \%$ of the female hormone estrogen secreted by the ovaries, adrenal zona fasciculata can also be part of estrogen secretion, use of animal removal of the ovaries, cut off the source of estrogen, caused by a sudden decrease in estrogen environment,can simulate the climacteric syndrome. The experiment uses the classic animal model, after modeling the animals showed typical menopausal endocrine disorders performance, levels of 5-HT, DA were significantly lower[4].

But studies have shown that, depression and substance information transfer in the brain 5-HT and DA are closely related, chronic stress can cause the rat content of frontal cortex, striatum, hypothalamus DA ware decreased, this may be a reward system functions under depression causes of damage to the central nervous system. Therefore, in this experiment, 5-HT, DA as a starting point to explore the mechanism of action of total flavonoids of Epimedium on menopausal depression [5]. Has obvious changes of sex hormones in menopausal women, mainly for elevated FSH and LH, E2 decreased, ovarian excretive estrogen mainly estradiol (E2), female three alcohol(E3) and 
estrone (E1), which is dominated by E2, its activity was also the highest[6]. Women in perimenopause due to declining ovarian function, gonadal function gradually decline, endocrine disorders, so that sex hormone FSH, LH and E2 levels change significantly. Women in menopause, due to decreased levels of E2, negative feedback effect on the pituitary gland, causing significantly increased pituitary FSH secretion; Corresponding decline in T levels, also contributed to the relative reduction of the amount converted to $\mathrm{E} 2$, causing the one hand of menopausal syndrome endocrine disorders [7].

The experimental results show that, total flavonoids of Epimedium can significantly improve the level of depression in rats horizontal - vertical motion score, brain homogenates levels of 5-HT and DA levels, improve thymus index, can be a very good improvement in depression model rat thymus, histopathological changes of hypothalamus. Provide experimental support for traditional Chinese medicine in the treatment of menopausal depression model and resource development and utilization of traditional Chinese medicine.

\section{References}

[1] Li Dong, Suiqi Gui,Wei Meng,etal. Establishment and evaluation of experimental animal model of Menopause Depression[J]. Chinese Journal of clinical rehabilitation,2004, 8(27):5871-5873.

[2] Ping Han,Zhuomin Song. Effect of solution liver Decoction modified on the nerve of rats with involutional depression recurrence 5-HT and blood lipid level[J]. Journal of clinical Chinese Medicine,2014,26 (4): :407-408.

[3] Ruiqi Li,Bai Ming, Mingsan Miao. Features and Prospect animal model of menopausal syndrome[J]. Journal of Traditional Chinese Medicine, 2012,27(169):708-710.

[4]Ruilai Xu. Laboratory study of qingre gengnian recipe in treating climacteric syndrome[J]. Modern preventive medicine,2011,38(5):866-868.

[5] Kai Xu,Xiuling Chen,Renhan Luo. Effeccts of Electroacupuncture at Sishencong (Ex-HN1) on 5-Hydroxytryptamine an Dopamine in Serum of Depression Patients[J]. Journal of New Chinese Medicine, 2011,43(4):87-89.

[6] Zeqin Gao. The main body of the female sex hormone[J]. Biology Teaching,2011,36(1): 69.

[7] Shuoyan Yu,Huili Liu,Mingsan Miao. Characteristics of the molecular mechanisms of menopausal syndrome and Chinese medicine treatment of menopausal syndrome[J]. Journal of Traditional Chinese Medicine, 2012,27(166):338-340. 